\title{
Querying the Absolute Need for either Faradic or Galvanic Stimulation in the Physical Treatment of Leprosy
}

\author{
JEAN (;ARI)INER \\ Sitanala Hospital, Tangarang. W'. Java, Indonesia
}

Electrical stimulation is commonly used in the re-education of muscles for two reasons, or in leprosy, perhaps three:

(1) For muscle testing, i.e. to trace which motor nerve or nerves are damaged, as following direct trauma, or as the result of a disease which affects the lower motor neurones indiscriminately, e.g. poliom yelitis.

(2) For stimulating a weak muscle to contract or a muscle whose motor nerve has been injured. This treatment is used only until a damaged motor nerve has recovered sufficiently so that the muscle can be self-activated. From this point, electrical stimulation is stopped and active exercise takes over. Only the latter can further increase muscle strength, electrical stimulation being a maintenance treatment only under this heading.

In leprosy, the lepra bacillus being so selective, the need for Reason No. 1, that is muscle testing to determine which nerve is damaged, is hardly necessary, the clinical signs being self-evident. As for Reason No. 2. in leprosy the cases in which electrical stimulation may be of value for a short period are those of tuberculoid leprosy, which are diagnosed before a peripheral nerve is damaged to any great extent. Unfortunately, many patients are inclined to wait far too long before coming for treatment to the leprosy clinics. In those cases in which an anti-leprosy drug has been given in time so that the nerve lesion remains partial and, it may be hoped, reversible, active exercise and the activities of daily living are the quickest way to strengthen weak muscles.

In the latter cases, the patients (particularly those in the 16 to 30 years age-group) frequently wish to return to their own social environment before their absence there has been noted and so are lost to further physical treatment. Again it is easy for them to exercise actively at home, provided they are taught a simple way of supporting the unstable joints while exercising, unt the muscles are strong enough for normal joint stability, at which stage their normal daily activities are sufficient to carry on the good work.

From this brief sketch it may be reasoned that in leprosy, re-education by the usual electrical stimulation is not essential, although a few patients are helped by it. Unfortunately, in leprosy a nerve lesion in an untreated case can progress so quickly that it is soon past the stage where electrical stimulation can be of any assistance.

The third situation in leprosy where electrical stimulation might be used is after reconstructive surgery. Here, it may be argued that such stimulation is 
unnecessary, even contra-indicated-although there may be one exception (mentioned below).

In reconstructive surgery of the hand or foot the muscle commonly used for transfer is normally strong and normally innervated. This means that from the beginning of post-operative re-education it will have the power of active movement (even after 3 weeks of joint immobilization in plaster of Paris); with active movement correctly maintained, no electrical stimulation is necessary.

Being a normally innervated muscle, it in itself does not need to re-learn to contract. It is the motor cortex which must recognize the new pattern of movement, so that the correct order may be given for the changed action of the muscle. It is found that with carefully correct and strict re-education it takes only one week for the cortex to master this new pattern. With this speed and accuracy, it seems unnecessary to use.electrical stimulation which, in effect, by-passes the motor cortex, the very place where the new recognition needs to be gained.

The exception (mentioned above) is that of a patient who, following reconstructive surgery, is not for some unfortunate reason taught the correct method of re-education (i.e. through the "mental pathway" of muscle action before its transferal). When this correct primary action is missing, a transferred muscle may be wasted, the new pattern of movement can be lost, and the normal muscle which has been transferred can atrophy from disuse. In this case, electrical stimulation may be able to pick up the muscle again, for a renewed attempt at correct re-education.

\section{Conclusion}

Without negating all possible good from electrical stimulation in leprosy-from the lack of essential need, and also taking into account the time spent in giving one treatment of electrical stimulation against the hundreds of patients waiting for help-it may be justifiable to say that it is not altogether warranted or necessary to include this special equipment in the leprosy clinic. 